\title{
Accurate Estimation of Breast Tumor Size: A Comparison Between Ultrasonography, Mammography, Magnetic Resonance Imaging, and Associated Contributing Factors
}

\author{
(1) Shilan Azhdeh ${ }^{1}$, (1) Ahmad Kaviani², (10) Nahid Sadighi ${ }^{1}$, (10 Maryam Rahmani ${ }^{1}$ \\ ${ }^{1}$ Department of Radiology, Tehran University of Medical Science, Tehran, Iran \\ ${ }^{2}$ Department of Surgery, Tehran University of Medical Science, Tehran, Iran
}

\begin{abstract}
Objective: This study aimed to provide further evidence on the accuracy of tumor size estimates and influencing factors.

Materials and Methods: In this cross-sectional study, patients with a biopsy-proven diagnosis of breast cancer referred to our hospital to obtain a preoperative magnetic resonance imaging (MRI) between 2015 and 2016 were included. Data from 76 breast cancer patients with 84 lesions were collected. All participants underwent ultrasonography and MRI, and their mammograms (MGMs) were reevaluated for tumor size estimation. Measurements by the three imaging modalities were compared with the pathologically determined tumor size to assess their accuracy. Influencing factors such as surgical management, molecular and histopathological subtypes, and Breast Imaging Reporting and Data System enhancement types in MRI were also assessed.

Results: The rates of concordance with the gold standard were $64.3 \%, 76.2 \%$, and $82.1 \%$ for MGM, ultrasound (US), and MRI measurements, respectively. Therefore, the highest concordance rate was observed in MRI-based estimates. Among the discordant cases, US and MGM underestimation were more prevalent (70\%); nevertheless, MRI showed significant overestimation (80\%). Tumor size estimates in patients whose MRIs presented with either non-mass enhancement $[\mathrm{p}=0.030$; odds ratio $(\mathrm{OR})=17.2 ; 95 \%$ confidence interval $(\mathrm{CI}): 1.3-225.9]$ or mass lesion with non-mass enhancement $(\mathrm{p}=0.001$; $\mathrm{OR}=51.0 ; 95 \% \mathrm{CI}$ : 5.0-518.4) were more likely to be discordant with pathological measurements compared with those in cases with only mass lesion on their MRIs.
\end{abstract}

Conclusion: MRI was more accurate than either US or MGM in estimating breast tumor size but had the highest overestimation rate. Therefore, caution should be practiced in interpreting data obtained from subjects whose MRIs present with non-mass enhancement or mass lesion with non-mass enhancement.

Keywords: Breast neoplasms, mammography, ultrasonography, magnetic resonance imaging, tumor size, molecular subtypes

Cite this article as: Azhdeh S, Kaviani A, Sadighi N, Rahmani M. Accurate Estimation of Breast Tumor Size: A Comparison Between Ultrasonography, Mammography, Magnetic Resonance Imaging, and Associated Contributing Factors. Eur J Breast Health 2021; 17(1): 53-61.

\section{Introduction}

Breast cancer is the second most common cause of cancer-related mortality in women worldwide, with a lifetime risk of approximately $12 \%$ (1). Tumor size is one of the main prognostic factors in breast cancer and is reported to correlate with lymph node involvement, tumor grade, and overall survival rate (2). Tumor size is also a factor assessed to determine treatment plans: breast conservation, mastectomy, or neoadjuvant chemotherapy (3).

Accordingly, precise estimation of tumor size is of utmost importance for planning a therapeutic strategy, and the main imaging modalities are mammogram (MGM), ultrasound (US), and magnetic resonance imaging (MRI). Each of these modalities has certain strengths and weaknesses in breast tumor evaluation. For instance, MGM is superior in identifying malignant calcifications; however, the obscurity of the margins and magnification variability limits the accuracy of measurements by this method (4). The sensitivity of MGM to detect malignant lesions in younger patients with dense breast tissue is also reported to be poor $(5,6)$. As for US, its ability to measure tumors in multiple planes is a great strength that enables a skilled operator to make measurements of its largest dimension (7). However, one main limitation of US is that it is highly operator dependent (8). MRI also offers the merit of multiplanar imaging along with a higher accuracy in assessing multicentric and multifocal lesions $(9,10)$; however, MRI has been reported to overestimate tumor size $(9,11)$, and the extent of background parenchymal enhancement $(\mathrm{BPE})$ affects its accuracy (12).

In this regard, studies have assessed the accuracy of tumor size estimation by MGM and US (11, 13-15), and compared their measurements with those by MRI $(10,16,17)$. In comparing US and MGM, some studies have reported that US has a higher accuracy than MGM $(7,11$, 
$15,18)$, whereas others found the opposite $(13,14,19)$. Among the studies that compared all three modalities, some reported MRI to have a higher accuracy $(16,20)$, whereas others found that MGM was more accurate $(21,22)$.

Considering the variability of the findings of the current literature on this subject, this study was designed to provide further evidence regarding the accuracy of MGM, US, and MRI in estimating breast tumor size by evaluating their concordance with the pathologically determined size of the surgical specimen and the effects of various factors on the accuracy of their measurements.

\section{Study design}

In this cross-sectional study, the target population included patients with a biopsy-proven diagnosis of breast cancer [Breast Imaging Reporting and Data System (BI-RADS) VI] (23), who were referred to Laleh Hospital in Tehran to obtain a preoperative MRI between 2015 and 2016. Indications for preoperative MRI in these patients included the following:

- Screening for presence of multifocal or multicentric lesions within the ipsilateral breast, for instance, in patients with invasive lobular carcinoma (ILC)

- Screening for involvement of the contralateral breast, for example, in patients with ductal carcinoma in situ (DCIS)

- Providing a more accurate evaluation of patients with dense breast composition, for example, candidates for breast-conserving surgery (BCS).

Patients who received neoadjuvant chemotherapy and subjects with gaps of longer than 1 month between their breast biopsy and MRI were excluded.

Based on these inclusion and exclusion criteria, eligible subjects were recruited through a convenience sampling method. All participants underwent ultrasonography and MRI, and their MGMs were also reevaluated. A breast specialist radiologist with more than 10 years of experience in the field performed the US assessment and evaluated the MGMs and MRIs of all patients.

\section{Magnetic resonance imaging}

Breast MRI was conducted using a dedicated surface breast coil of a Siemens Avanto 1.5 Tesla MRI scanner (Siemens Healthcare, Erlangen, Germany) with the patient lying in a prone position. Standard sequences were obtained, including an axial turbo inversion recovery magnitude (TR/TE $5600 / 59 \mathrm{~ms}$; a flip angle of $142^{\circ}$; viewfield of 340 $\mathrm{mm}$; matrix size of $314 \times 320$; slice thickness of $4 \mathrm{~mm}$; acquisition time of $2 \mathrm{~min}$ and $55 \mathrm{~s}$ ), an axial nonfat suppressed T1-weighted flash 3D (TR/TE 8.6/4.7; a flip angle of $20^{\circ}$; viewfield of $340 \mathrm{~mm}$; matrix size of $323 \times 448$; slice thickness of $1 \mathrm{~mm}$; acquisition time of $1 \mathrm{~min}$ and $45 \mathrm{~s}$ ), and axial T1-weighted flash $3 \mathrm{D}$ pre-contrast and post-contrast sequences (TR/TE 6/1.69 ms; a flip angle of $10^{\circ}$; viewfield of $340 \mathrm{~mm}$; matrix size of $342 \times 384$; slice thickness of $1.6 \mathrm{~mm}$; acquisition time of $7 \mathrm{~min}$ and $36 \mathrm{~s}$ ). Six post-contrast dynamic sequences with 55-s intervals starting at $20 \mathrm{~s}$ were obtained. Intravenous administration of $0.1 \mathrm{mmol} / \mathrm{L}$ gadopentetate dimeglumine (Magnevist, Bayer, Germany) was used as the contrast in this protocol. T1 contrastenhanced subtraction images were used for visual evaluation and categorization of BPE into four levels, namely, minimal, mild,
(24). Lesions were classified into three groups according to their MRI image appearance including mass lesions, non-mass enhancements, and mass lesions with non-mass components. The maximum diameter of the tumor was measured on the second subtraction post-contrast sequences and recorded in millimeters.

\section{Ultrasonography}

The same radiologist, who was blinded to the MRI results, performed US assessment using a digital ultrasound scanner (Phillips iU22 Manufactured by Philips Ultrasound Bothell-Everett Highway Bothell, WA 98021-8431 USA) equipped with a 6-14 Megahertz linear probe. The size of the tumor was determined at its greatest dimension and recorded for statistical analyses.

\section{Mammography}

Patients' MGMs obtained using full-field digital mammography unit were reevaluated in both the craniocaudal and mediolateral-oblique views. Patients were categorized into three groups, namely, mass, microcalcification, and distortion, based on the main characteristic of their lesions on MGMs. The maximum size of the lesion was also measured on the images and recorded in millimeters.

\section{Histopathological assessments}

The final pathology of the tumor and its grade were recorded on the basis of post-surgical evaluations. Standard immunohistochemical methods were used to determine the positivity of the tumor for estrogen receptor (ER), progesterone receptor (PR), human epidermal growth factor receptor 2 (HER2), and Ki-67 protein level. Based on the results of these assessments, ER-positive, PR-positive, and HER2-negative tumors were classified as luminal A molecular subtype, whereas ER-positive, PR-positive, and either HER2positive or high $\mathrm{Ki}-67$ level lesions were categorized as luminal B (25). HER2 overexpression was also defined as the specimen positive for HER $2>30 \%$ of invasive tumor cells $(3+)$ but negative for both ER and PR. Finally, the pathologic tumor size was also measured by a breast pathologist, and the greatest lesion diameter was recorded in millimeters for analyses.

\section{Statistical analysis}

All analyses were conducted using SPSS software for Windows, version 22 (IBM Corp, Armonk, NY, USA) (26). Primary descriptive statistics of the study were reported as frequency distribution, mean, and standard deviation. Concordance between the measurements made by each of the three imaging modalities with the pathologically determined tumor size was evaluated using a cut-off point of $5 \mathrm{~mm}$ according to previous studies (27-30).

The effects of various factors were evaluated on the accuracy of tumor size measurement using the three imaging techniques. To evaluate the correlation between qualitative variables, chi-squared and Fisher's Exact tests were used as needed. To determine the independent risk factors for discordance between measurements, multivariate logistic regression analysis was performed. The variables found to be significantly correlated with the discordance of the measurements in univariate analysis were included in the regression models. A p-value of less than 0.05 was considered statistically significant in all analyses.

\section{Ethical considerations}

The objectives and methods of the study were thoroughly explained to the patients, and informed written consent was obtained from 
all subjects willing to participate in the study. They were reassured that their inclusion in the survey would not affect their treatment in any way and that they could withdraw from the study at any time. Data gathered from patients were considered confidential and used anonymously, and only the main researchers had access to the information. The study protocol was evaluated and approved by the Institutional Review Board of the Tehran University of Medical Sciences.

\section{Results}

\section{Descriptive statistics}

A total of 86 breast cancer patients were recruited to participate in the study, of which 10 received neoadjuvant chemotherapy before surgical resection of the tumor and were subsequently excluded. Data from 76 patients with 84 lesions in their breasts were analyzed in the study. Descriptive statistics for variables of interest are presented in Table 1.

Most participants (67.9\%) underwent BCS and 27 (32.1\%) mastectomy. The most common pathology type in these patients was a combination of DCIS and invasive ductal carcinoma (IDC) $(42.9 \%)$; $27(32.1 \%)$ lesions were reported to be IDC, 16 (19.0\%) ILC, and 4 (4.8\%) DCIS alone.

Based on the results of immunohistochemical assessments, HER2 overexpression was reported in 12 cases (14.3\%), and 16 (19.0\%) were found to be triple negative, and the molecular subtype was luminal A in 24 lesions (28.6\%) and luminal B in 33 (39.3\%).

As for the findings of imaging modalities, no particular lesions were observed in 13 MGMs (15.5\%). The lesion was visualized as a mass in $46 \mathrm{MGMs}(54.8 \%)$ and as distortion in $16(19.0 \%)$, whereas microcalcifications were observed in nine MGMs (10.7\%). Breast composition in most cases (51.2\%) was reported as C, whereas in $22(26.2 \%), 16(19.0 \%)$, and $3(3.6 \%)$ cases were $\mathrm{D}, \mathrm{B}$, and $\mathrm{A}$, respectively. However, the lesion was visualized as a mass in 74 (88.1\%) of the evaluated MRIs, non-mass enhancement in $3(3.6 \%)$, and both mass lesions and non-mass components in 7 (8.3\%). Based on the MRIs of these cases, BPE was also reported to be minimal in $2(2.4 \%)$, mild in $24(28.6 \%)$, moderate in $29(34.5 \%)$, and marked in 29 (34.5\%).

Table 2 presents the overall statistics of the measurements of the three imaging modalities and pathological assessments. According to these findings, MGM measurements were concordant with pathologically determined tumor sizes in 54 lesions (64.3\%). Among the $30(35.7 \%)$ discordant cases, underestimation (70.0\%) was more prevalent than overestimation (30.0\%). As for the US, the estimates were concordant with the gold standard in 64 cases $(76.2 \%)$, with $80 \%$ of the discordant measurements being underestimates and $20 \%$ overestimates. The highest concordance rate was observed in MRIbased estimates $(82.1 \%)$ with only 15 cases showing discordance, which is composed of underestimates in three cases (20\%) and overestimates in $12(80.0 \%)$.

\section{Analytical statistics}

The correlation between the accuracy of tumor size measurements by each of the three imaging modalities was evaluated for all variables included in the study. Accordingly, Tables 3, 4, and 5 present the results of these analyses for MGM, US, and MRI, respectively.

Table 1. Descriptive statistics of evaluated variables in the study

Variables

Age group (years)

$<50$

$\geq 50$

Positive family history

Palpability

Non-palpable

Palpable

Surgical management

BCS

57 (67.9)

Mastectomy

$27(32.1)$

\section{Pathology}

DCIS

IDC

$\mathrm{DCIS}+\mathrm{IDC}$

ILC

$16(19.0)$

Other

$1(1.2)$

Grade

I

$9(10.7)$

II

$52(61.9)$

III

$23(27.4)$

Locality

Single lesion

$55(65.5)$

Multifocal

Multicentric

2 (2.4)

Histopathological assessments

ER positive

57 (67.9)

PR positive

57 (67.9)

HER2 positive

$22(26.2)$

HER2 overexpression

$12(14.3)$

Triple negative

$16(19.0)$

Luminal A

24 (28.6)

Luminal B

$33(39.3)$

\section{Appearance on MGM}

Mass

$46(64.8)$

Microcalcification

9 (12.7)

Distortion

$16(22.5)$

Breast composition on MGM

\section{A}

B

16 (19.0)

C

$43(51.2)$

D

$22(26.2)$ 
Table 1. Continued

\begin{tabular}{lc} 
Variables & Frequency (\%) \\
\hline MRI enhancement & \\
Mass & $74(88.1)$ \\
Non-mass & $3(3.6)$ \\
Mass with non-mass components & $7(8.3)$ \\
BPE on MRI & \\
Minimal & $2(2.4)$ \\
Mild & $24(28.6)$ \\
Moderate & $29(34.5)$ \\
Marked & $29(34.5)$
\end{tabular}

BCS: Breast-conserving surgery; DCIS: Ductal carcinoma in situ; IDC: Invasive ductal carcinoma; ILC: Invasive lobular carcinoma; ER: Estrogen receptor; PR: Progesterone receptor; HER2: Human epidermal growth factor receptor 2; MGM: Mammogram; MRI: Magnetic resonance imaging; BPE: Background parenchymal enhancement

As can be seen, none of the included variables were significantly correlated with the type of discordance between MGM and pathological measurements. Univariate analyses showed that the surgical management type $(\mathrm{p}=0.002)$, tumor pathology $(\mathrm{p}=0.007)$, and lesion locality $(\mathrm{p}=0.006)$ were significantly correlated with the accuracy of MGM measurements. Subsequently, these variables were included in a regression model, and the results of which showed that tumor size estimates via MGM in patients that underwent mastectomy were more likely to be discordant with the pathological measurements compared with that in subjects who underwent BCS [p=0.025; odds ratio (OR): 4.3; 95\% confidence interval (CI): 1.2-15.4].

As presented in Table 4, univariate analysis found that surgical management type $(\mathrm{p}<0.001)$ and lesion pathology $(\mathrm{p}=0.039)$ were significantly correlated with the accuracy of US. A regression model including these two variables showed that tumor size estimation via US in cases that underwent mastectomy was more likely to be discordant with the pathological measurements compared with that in patients who underwent BCS ( $\mathrm{p}=0.006$; OR: 5.7; 95\% CI: 1.7-19.3).

According to the results presented in Table 5, underestimation of tumor size by MRI was more prevalent in patients with HER2 overexpression $(\mathrm{p}=0.024)$. Univariate analysis found that MRI enhancement type $(\mathrm{p}<0.001)$ was significantly correlated with MRI accuracy. The surgical management type also had a borderline p-value of 0.053 , and both of these variables were included in the regression model. This analysis showed that tumor size estimates in patients whose MRIs showed either non-mass enhancement ( $\mathrm{p}=0.030$; OR: 17.2 ; 95\% CI: 1.3-225.9) or mass lesion with non-mass enhancement ( $\mathrm{p}=0.001$; OR: 51.0; 95\% CI: 5.0-518.4) were more likely to be discordant with pathological measurements compared with that in cases with only mass lesions on their MRIs (Figures 1 and 2).

\section{Discussion and Conclusion}

This study provided further evidence on the accuracy of MGM, US, and MRI in estimating breast tumor size by evaluating their concordance with the gold standard of pathological measurements of the surgical
Table 2. Overall statistics of the measurements by the three imaging modalities and pathological assessments

\begin{tabular}{|lccccc|}
\hline Tumor size & $\mathbf{n}$ & Minimum & Maximum & Mean & SD \\
\hline Pathology & 84 & 5 & 80 & 22.29 & 13.195 \\
MGM & 84 & 0 & 80 & 18.87 & 13.913 \\
US & 84 & 0 & 80 & 18.26 & 10.648 \\
MRI & 84 & 6 & 84 & 24.74 & 16.134 \\
\hline
\end{tabular}

MGM: Mammogram; US: Ultrasound; MRI: Magnetic resonance imaging; SD: Standard deviation; n: Number

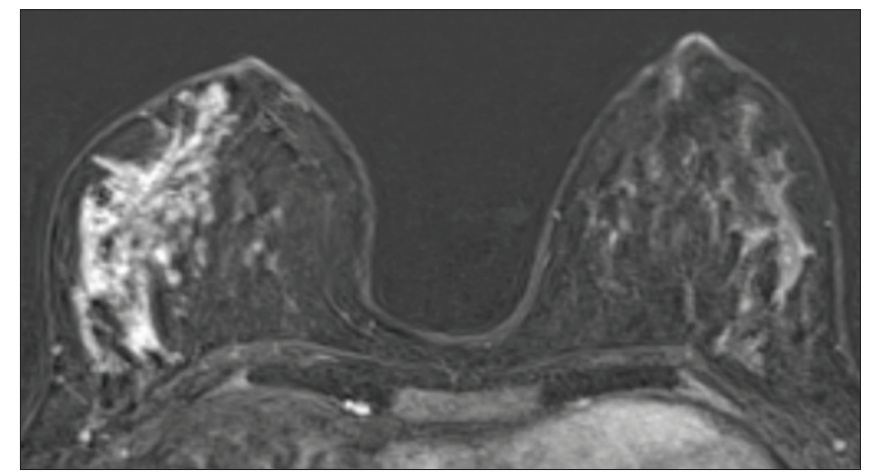

Figure 1. Non-mass enhancement. Magnetic resonance imaging of non-mass enhancement in a 30-year-old patient with invasive ductal carcinoma and extensive ductal carcinoma in situ component. The nonmass enhancement measured $76 \mathrm{~mm}$ along the maximum diameter, whereas the pathology reported $11 \mathrm{~mm}$ invasive component along with $55 \mathrm{~mm}$ in situ component. MRI inaccurately measured the tumor size in this non-mass enhancement

MRI: Magnetic resonance imaging

accuracy of their measurements, including histology type, molecular subtypes, breast density, BI-RADS type of enhancement, and BPE.

Statistical analysis of data gathered from 84 lesions in 76 breast cancer patients found that the rates of concordance with the gold standard were $64.3 \%, 76.2 \%$, and $82.1 \%$ for MGM, US, and MRI measurements, respectively. The proportion of discordant cases that were reported to be an overestimation of the actual tumor size was $30 \%$ for MGM, 20\% for US, and $80.0 \%$ for MRI.

As mentioned, many studies have evaluated the accuracy of tumor size estimates from these three imaging modalities with contradictory results. For instance, some studies have reported US to be more accurate than $\operatorname{MGM}(7,11,15,18)$, whereas others have reported the opposite $(13,14,21,31)$. Boetes et al. (20) analyzed histologic results and imaging findings of 61 tumors in 60 women who had mastectomies and reported MRI to have the highest accuracy among the three imaging modalities, with MGM and US underestimating tumor sizes by $14 \%$ and $18 \%$, respectively. Meanwhile, Gruber et al. (21) analyzed data from 121 patients with primary breast cancer and reported that US significantly underestimated tumor size. The study further revealed that MRI overestimated lesion dimensions, but the differences were not significant, whereas MGM showed the most accurate measurements with no significant difference with histological sizing (21). In a more extensive survey conducted on 
6,543 patients with unifocal, unilateral primary breast cancer, Stein et al. (32) reported a slightly higher correlation between MGM and pathological examination than US ( $\mathrm{r}=0.61$ vs 0.60 , respectively).

Further analyses showed that tumor size estimation with either of the two imaging modalities of MGM and US in patients who underwent mastectomy was more likely to be discordant with pathological measurements compared with that in subjects who underwent BCS. These findings could be attributed to underestimate tumor size in

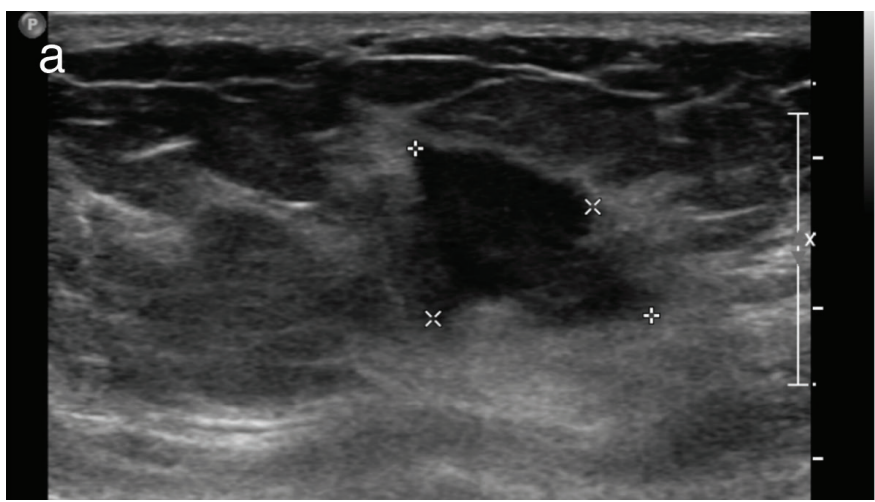

mastectomy specimens by pathology. Just as Rominger et al. (33) explained, mastectomy specimens are sliced and evaluated along the anatomical axis, not the tumor axis. Accordingly, they suggested taking advantage of preoperative MRI for determining the axis, along which the tumor should be sliced for pathological evaluations. For MRI, owing to its overall higher accuracy for all lesions, the correlation between the type of surgical intervention and concordance with the gold standard loses its significance, and the pattern of enhancement becomes more prominent. In this regard, the analyses showed that

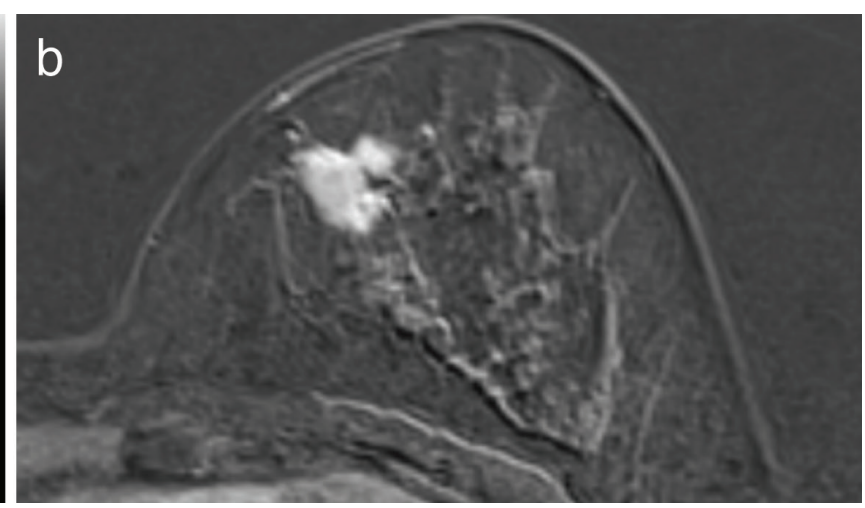

Figure 2. Concordant mass in magnetic resonance imaging (MRI) and ultrasound (US). A 32-year-old patient with invasive ductal carcinoma measuring $22 \mathrm{~mm}$ in the pathology (a). US shows an irregular mass measuring $19 \mathrm{~mm}$ in the upper inner quadrant of the left breast (b). MRI shows lobulated- enhancing mass with $22 \mathrm{~mm}$ in the longest dimension. In this patient, MRI and US are in concordance with the pathology

Table 3. Correlation between the evaluated factors in the study with accuracy of tumor size estimation via MGM

\begin{tabular}{|c|c|c|c|c|c|c|c|}
\hline \multirow{2}{*}{ Variables } & \multirow{2}{*}{$\begin{array}{c}\text { Concordant } \\
(n=54)\end{array}$} & \multicolumn{2}{|c|}{$\begin{array}{l}\text { Discordant } \\
(n=30)\end{array}$} & \multirow{2}{*}{$p^{a}$} & \multirow{2}{*}{$P^{b}$} & \multirow{2}{*}{$P^{c}$} & \multirow{2}{*}{$\begin{array}{c}\text { Adjusted odds } \\
\text { ratio } \\
(95 \% \mathrm{Cl})\end{array}$} \\
\hline & & $\begin{array}{l}\text { Underestimation } \\
\qquad(n=21)\end{array}$ & $\begin{array}{l}\text { Overestimation } \\
\qquad(n=9)\end{array}$ & & & & \\
\hline \multicolumn{8}{|c|}{ Age group (years) } \\
\hline$<50$ & $38(61.3 \%)$ & $17(27.4 \%)$ & $7(11.3 \%)$ & \multirow{2}{*}{0.842} & \multirow{2}{*}{0.336} & - & - \\
\hline$\geq 50$ & $16(72.7 \%)$ & $4(18.2 \%)$ & $2(9.1 \%)$ & & & - & - \\
\hline \multicolumn{8}{|c|}{ Surgical management } \\
\hline BCS & $43(75.4 \%)$ & $10(17.5 \%)$ & $4(7.0 \%)$ & \multirow{2}{*}{0.873} & \multirow{2}{*}{0.002} & - & Reference \\
\hline Mastectomy & $11(40.7 \%)$ & $11(40.7 \%)$ & $5(18.5 \%)$ & & & 0.025 & $4.3(1.2-15.4)$ \\
\hline \multicolumn{8}{|l|}{ Pathology } \\
\hline DCIS & $3(75.0 \%)$ & $1(25.0 \%)$ & $0(0.0 \%)$ & & & - & Reference \\
\hline IDC & $21(77.8 \%)$ & $4(14.8 \%)$ & $2(7.4 \%)$ & & & 0.938 & - \\
\hline DCIS + IDC & $25(69.4 \%)$ & 7 (19.4\%) & $4(11.1 \%)$ & 0.846 & 0.007 & 0.593 & - \\
\hline ILC & $4(25.0 \%)$ & $9(56.3 \%)$ & $3(18.8 \%)$ & & & 0.119 & - \\
\hline Other & $1(100.0 \%)$ & $0(0.0 \%)$ & $0(0.0 \%)$ & & & 1.000 & - \\
\hline \multicolumn{8}{|l|}{ Locality } \\
\hline Single lesion & 41 (74.5\%) & $11(20.0 \%)$ & $3(5.5 \%)$ & & & - & Reference \\
\hline Multifocal & $11(40.7 \%)$ & $10(37.0 \%)$ & $6(22.2 \%)$ & 0.338 & 0.006 & 0.302 & - \\
\hline Multicentric & 2 (100.0\%) & $0(0.0 \%)$ & $0(0.0 \%)$ & & & 0.999 & - \\
\hline
\end{tabular}

ap of significant difference between underestimation versus overestimation by chi-square test and as needed Fisher's Exact test, bP of significant difference between concordant versus discordant by chi-square test and as needed Fisher's Exact test, cP of significant difference between concordant versus discordant by multivariable logistic regression.

MGM: Mammogram; CI: Confidence interval; BCS: Breast-conserving surgery; DCIS: Ductal carcinoma in situ; IDC: Invasive ductal carcinoma; ILC: Invasive lobular carcinoma; n: Number

$\mathrm{p}$-values in bold was considered statistically significant 


\section{Table 4. Correlation between the evaluated factors in the study with accuracy of tumor size estimation via US}

\begin{tabular}{|c|c|c|c|c|c|c|c|}
\hline \multirow{2}{*}{ Variables } & \multirow{2}{*}{$\begin{array}{l}\text { Concordant } \\
\qquad(n=64)\end{array}$} & \multicolumn{2}{|c|}{$\begin{array}{l}\text { Discordant } \\
\qquad(n=20)\end{array}$} & \multirow{2}{*}{$P^{a}$} & \multirow{2}{*}{$P^{b}$} & \multirow{2}{*}{$P^{c}$} & \multirow{2}{*}{$\begin{array}{l}\text { Adjusted odds ratio } \\
(95 \% \mathrm{Cl})\end{array}$} \\
\hline & & $\begin{array}{l}\text { Underestimation } \\
\qquad(n=16)\end{array}$ & $\begin{array}{l}\text { Overestimation } \\
\qquad(n=4)\end{array}$ & & & & \\
\hline \multicolumn{8}{|c|}{ Age group (years) } \\
\hline$<50$ & 45 (72.6\%) & $15(24.2 \%)$ & $2(3.2 \%)$ & \multirow{2}{*}{0.028} & \multirow{2}{*}{0.192} & - & - \\
\hline$\geq 50$ & 19 (86.4\%) & $1(4.5 \%)$ & $2(9.1 \%)$ & & & - & - \\
\hline \multicolumn{8}{|c|}{ Surgical management } \\
\hline $\mathrm{BCS}$ & $50(87.7 \%)$ & $5(8.8 \%)$ & $2(3.5 \%)$ & \multirow{2}{*}{0.482} & \multirow{2}{*}{$<0.001$} & \multirow{2}{*}{0.006} & Reference \\
\hline Mastectomy & 14 (51.9\%) & $11(40.7 \%)$ & $2(7.4 \%)$ & & & & $5.7(1.7-19.3)$ \\
\hline \multicolumn{8}{|l|}{ Pathology } \\
\hline DCIS & 2 (50.0\%) & $2(50.0 \%)$ & $0(0.0 \%)$ & & & \multirow{2}{*}{0.151} & Reference \\
\hline IDC & 23 (85.2\%) & $3(11.1 \%)$ & $1(3.7 \%)$ & & & & - \\
\hline DCIS + IDC & 30 (83.3\%) & $5(13.9 \%)$ & $1(2.8 \%)$ & & & 0.358 & - \\
\hline ILC & $8(50.0 \%)$ & $6(37.5 \%)$ & $2(12.5 \%)$ & 0.866 & 0.039 & 0.843 & - \\
\hline Other & $1(100.0 \%)$ & $0(0.0 \%)$ & $0(0.0 \%)$ & & & 1.000 & - \\
\hline
\end{tabular}

ap of significant difference between underestimation versus overestimation by chi-square test and as needed Fisher's Exact test, bP of significant difference between concordant versus discordant by chi-square test and as needed Fisher's Exact test, cP of significant difference between concordant versus discordant by multivariable logistic regression.

US: Ultrasound; Cl: Confidence interval; BCS: Breast-conserving surgery; DCIS: Ductal carcinoma in situ; IDC: Invasive ductal carcinoma; ILC: Invasive lobular carcinoma; n: Number

p-values in bold was considered statistically significant

tumor size estimation in patients whose MRIs presented with either non-mass enhancement or mass lesion with non-mass enhancement was more likely to be discordant with the pathological measurements compared with that in cases with only mass lesion on their MRIs.

Rominger et al. (33) also found that the only factor that significantly predicts discordance between MRI and histological measurements is non-mass enhancement of the lesion. Their findings were congruent with the results of this study; however, in our study, the number of non-mass enhancement and mass lesions with non-mass enhancement was limited.

In another study, Baek et al. (34) showed that HER2 overexpression along with BPE could affect the accuracy of measurements using MRI; however, the most important factor that contributed to the discordance of MRI measurements with that of the pathological evaluations is the BI-RADS enhancement type. Our study also showed that HER2 overexpression is related to the underestimation of tumor size in MRI $(\mathrm{p}=0.024)$, but the most significant attributing factor was the lesion enhancement type (mass vs non-mass).

Previous studies have reported that MGM and US tend to underestimate the size of ILCs. In our series, 19\% of the lesions were diagnosed as ILC, and the rates of concordance for US and MGM were $50 \%$ and $25 \%$, respectively, whereas MRI provided a concordance rate of $68 \%$. Therefore, congruent with previous studies, our results showed that MRI is more accurate for tumor size measurement in ILC subtype lesions, and the accuracy of this modality is less affected by the
Overall, despite the higher accuracy of MRI compared with that of US and MGM in estimating breast tumor sizes, the high cost, higher overestimation rate, and limited availability have prevented widespread application of this imaging modality in standard practice. In this regard, it seems that MRI should be reserved for specific subject groups categorized as high risk by the American Cancer Society. Caution should be practiced in interpreting data obtained from subjects whose MRIs present with non-mass enhancement, since tumor size could be overestimated by MRI in these subgroups.

Although the limited sample population included in this survey could have affected the results of our analyses, the specific setting of this study enabled us to gather information from the three imaging modalities in all our subjects, which minimized missing data in the analyses. Reevaluation of the patients' MGMs along with their USs and MRIs by a single breast specialist radiologist with extensive experience in the field decreased interobserver variability to its minimum; however, this is noted as a limitation because of the possibility of intraobserver error. Further investigations are required to determine the factors associated with tumor size estimation discordance via imaging modalities with pathological measurements.

In conclusion, MRI was more accurate than US and MGM in estimating breast tumor size with concordance rates of $82.1 \%, 76.2 \%$, and $64.3 \%$ respectively, but it had the highest overestimation rate (80\%) among the three modalities. Thus, caution should be practiced in interpreting data obtained from subjects whose MRIs present with non-mass enhancement or mass lesion with non-mass. 
Table 5. Correlation between the evaluated factors in the study with accuracy of tumor size estimation via MRI

\begin{tabular}{|c|c|c|c|c|c|c|c|}
\hline \multirow{2}{*}{ Variables } & \multirow{2}{*}{$\begin{array}{l}\text { Concordant } \\
\qquad(n=69)\end{array}$} & \multicolumn{2}{|c|}{$\begin{array}{l}\text { Discordant } \\
\quad(n=15)\end{array}$} & \multirow{2}{*}{$p^{a}$} & \multirow{2}{*}{$P^{b}$} & \multirow{2}{*}{$P^{c}$} & \multirow{2}{*}{$\begin{array}{c}\text { Adjusted odds } \\
\text { ratio } \\
(95 \% \mathrm{Cl})\end{array}$} \\
\hline & & $\begin{array}{l}\text { Underestimation } \\
\qquad(n=3)\end{array}$ & $\begin{array}{l}\text { Overestimation } \\
\qquad(n=12)\end{array}$ & & & & \\
\hline \multicolumn{8}{|l|}{ Age group (years) } \\
\hline$<50$ & 49 (79.0\%) & $2(3.2 \%)$ & $11(17.7 \%)$ & \multirow{2}{*}{0.255} & \multirow{2}{*}{0.211} & - & - \\
\hline$\geq 50$ & 20 (90.9\%) & $1(4.5 \%)$ & $1(4.5 \%)$ & & & - & - \\
\hline \multicolumn{8}{|l|}{ Surgical management } \\
\hline BCS & $50(87.7 \%)$ & $1(1.8 \%)$ & $6(10.5 \%)$ & \multirow{2}{*}{0.605} & \multirow{2}{*}{0.053} & \multirow{2}{*}{0.697} & Reference \\
\hline Mastectomy & $19(70.4 \%)$ & $2(7.4 \%)$ & $6(22.2 \%)$ & & & & - \\
\hline \multicolumn{8}{|c|}{ Histopathological findings } \\
\hline ER positive & $48(84.2 \%)$ & $1(1.8 \%)$ & $8(14.0 \%)$ & 0.292 & 0.472 & - & - \\
\hline PR positive & $48(84.2 \%)$ & $1(1.8 \%)$ & $8(14.0 \%)$ & 0.292 & 0.472 & - & - \\
\hline HER2 positive & $18(81.8 \%)$ & $2(9.1 \%)$ & $2(9.1 \%)$ & 0.080 & 0.963 & - & - \\
\hline HER2 overexpression & $9(75.0 \%)$ & $2(16.7 \%)$ & $1(8.3 \%)$ & 0.024 & 0.485 & - & - \\
\hline Triple negative & $13(81.3 \%)$ & $0(0.0 \%)$ & $3(18.8 \%)$ & 0.333 & 0.917 & - & - \\
\hline Luminal A & $21(87.5 \%)$ & $1(4.2 \%)$ & $2(8.3 \%)$ & 0.519 & 0.417 & - & - \\
\hline Luminal B & $27(81.8 \%)$ & $0(0.0 \%)$ & $6(18.2 \%)$ & 0.114 & 0.950 & - & - \\
\hline \multicolumn{8}{|l|}{ MRI enhancement } \\
\hline Mass & $67(90.5 \%)$ & $2(2.7 \%)$ & $5(6.8 \%)$ & & & & Reference \\
\hline Non-mass & $1(33.3 \%)$ & $0(0.0 \%)$ & $2(66.7 \%)$ & 0.650 & $<0.001$ & 0.030 & $\begin{array}{c}17.2 \\
(1.3-225.9)\end{array}$ \\
\hline Mass + non-mass & $1(14.3 \%)$ & $1(14.3 \%)$ & $5(71.4 \%)$ & & & 0.001 & $\begin{array}{c}51.0 \\
(5.0-518.4)\end{array}$ \\
\hline \multicolumn{8}{|l|}{ BPE on MRI } \\
\hline Minimal & $2(100.0 \%)$ & $0(0.0 \%)$ & $0(0.0 \%)$ & & & - & - \\
\hline Mild & $21(87.5 \%)$ & $1(4.2 \%)$ & $2(8.3 \%)$ & & & 一 & - \\
\hline Moderate & $24(82.8 \%)$ & $1(3.4 \%)$ & $4(13.8 \%)$ & 0.788 & 0.639 & - & - \\
\hline Marked & 22 (75.9\%) & $1(3.4 \%)$ & $6(20.7 \%)$ & & & - & - \\
\hline
\end{tabular}

ap of significant difference between underestimation versus overestimation by chi-square test and as needed Fisher's Exact test, bP of significant difference between concordant versus discordant by chi-square test and as needed Fisher's Exact test, cP of significant difference between concordant versus discordant by multivariable logistic regression.

MRI: Magnetic resonance imaging; Cl: Confidence interval; BCS: Breast-conserving surgery; ER: Estrogen receptor; PR: Progesterone receptor; HER2: Human epidermal growth factor receptor 2; BPE: Background parenchymal enhancement; n: Number p-values in bold was considered statistically significant

\section{Key Points}

- $\quad$ MRI is the most accurate imaging technique in estimating breast tumor size.

- MRI is more accurate for tumor size measurement in ILC subtype lesions.

- HER2 overexpression is related to underestimation of tumor size in MRI.

Ethics Committee Approval: The study protocol was evaluated and approved by the Institutional Review Board of the Tehran University of Medical Sciences (no: IR.TUMS.VCR.REC.1395.604, date: 2016/9/13).
Informed Consent: The objectives and methods of the study were thoroughly explained to the patients, and informed written consent was obtained from all subjects willing to participate in the study.

Peer-review: Externally-peer reviewed.

\section{Authorship Contributions}

Concept: S.A., M.R.; Design: S.A., M.R., A.K.; Supervision: M.R., N.S., A.K.; Funding: M.R.; Materials: S.A., M.R., A.K.; Data Collection and/or Processing: S.A., M.R., N.S., A.K.; Analysis and/or Interpretation: S.A., M.R., N.S., A.K.; Literature Review: S.A.; Writing: S.A., N.S.; Critical Review: M.R., A.K.

Conflict of Interest: No conflict of interest was declared by the authors.

Financial Disclosure: The authors did not receive any financial support or grant. 


\section{References}

1. U.N.I.o. Health, National Cancer Institute. Surveillance, Epidemiology, and End Results Program. National Cancer Institute. 2011; Available at: https://seer.cancer.gov/statfacts/html/breast.html

2. Szabó BK, Aspelin P, Wiberg MK, Tot T, Boné B. Invasive breast cancer: correlation of dynamic MR features with prognostic factors. Eur Radiol 2003; 13: 2425-2435. (PMID: 12898176) [Crossref]

3. Houssami N, Ciatto S, Macaskill P, Lord SJ, Warren RW, Dixon JM. Accuracy and surgical impact of magnetic resonance imaging in breast cancer staging: systematic review and meta-analysis in detection of multifocal and multicentric cancer. J Clin Oncol 2008; 26: 3248-3258. PMID: 18474876 [Crossref]

4. Feig S. Breast masses, Mammographic and sonographic evaluation. Radiol Clin North Am 1992; 30: 67-92. (PMID: 1732936) [Crossref]

5. Yang WT, Lam W, Cheung H, Suen M, King W, Metreweli C. Sonographic, magnetic resonance imaging, and mammographic assessments of preoperative size of breast cancer. J Ultrasound Med 1997; 16: 791-797. (PMID: 9401992) [Crossref]

6. Fornage BD, Toubas O, Morel M. Clinical, mammographic, and sonographic determination of preoperative breast cancer size. Cancer 1987; 60: 765-771. (PMID: 3297295) [Crossref]

7. Madjar H, Ladner H, Sauerbrei W, Oberstein A, Prömpeler H, Pfleiderer A. Preoperative staging of breast cancer by palpation, mammography and high-resolution ultrasound. Ultrasound Obstet Gynecol 1993; 3: 185190. (PMID: 14533601) [Crossref]

8. Farina R, Sparano A. Errors in sonography. In: Errors in Radiology. Milano:Springer; 2012. p. 79-85.

9. Lehman CD, Gatsonis C, Kuhl CK, Hendrick RE, Pisano ED, Hanna L. MRI evaluation of the contralateral breast in women with recently diagnosed breast cancer. N Engl J Med 2007; 356: 1295-1303. (PMID: 17392300) [Crossref]

10. Berg WA, Gutierrez L, NessAiver MS, Carter WB, Bhargavan M, Lewis RS, et al. Diagnostic accuracy of mammography, clinical examination, US, and MR imaging in preoperative assessment of breast cancer. Radiology 2004; 233: 830-849. (PMID: 15486214) [Crossref]

11. Hieken TJ, Harrison J, Herreros J, Velasco JM. Correlating sonography, mammography, and pathology in the assessment of breast cancer size. Am J Surg 2001; 182: 351-354. (PMID: 11720669) [Crossref]

12. Iacconi C, Thakur SB, Dershaw DD, Brooks J, Fry CW, Morris EA. Impact of fibroglandular tissue and background parenchymal enhancement on diffusion weighted imaging of breast lesions. Eur J Radiol 2014; 83: 2137-2143. (PMID: 25445896) [Crossref]

13. Golshan M, Fung B, Wiley E, Wolfman J, Rademaker A, Morrow M. Prediction of breast cancer size by ultrasound, mammography and core biopsy. Breast 2004; 13: 265-271. (PMID: 15325659) [Crossref]

14. Heusinger K, Löhberg C, Lux M, Papadopoulos T, Imhoff K, SchulzWendtland R. Assessment of breast cancer tumor size depends on method, histopathology and tumor size itself. Breast Cancer Res Treat 2005; 94: 17-23. (PMID: 16142441) [Crossref]

15. Shoma A, Moutamed A, Ameen M, Abdelwahab A. Ultrasound for accurate measurement of invasive breast cancer tumor size. Breast J 2006; 12: 252-256. (PMID: 16684323) [Crossref]

16. Wasif N, Garreau J, Terando A, Kirsch D, Mund DF, Giuliano AE. MRI versus ultrasonography and mammography for preoperative assessment of breast cancer. Am Surg 2009; 75: 970-975. (PMID: 19886147) [Crossref]

17. Lim HI, Choi JH, Yang JH, Han BK, Lee JE, Lee SK. Does pre-operative breast magnetic resonance imaging in addition to mammography and breast ultrasonography change the operative management of breast carcinoma?. Breast Cancer Res Treat 2010; 119: 163. (PMID: 19760039)
18. Fasching PA, Heusinger K, Loehberg CR, Wenkel E, Lux MP, Schrauder $\mathrm{M}$. Influence of mammographic density on the diagnostic accuracy of tumor size assessment and association with breast cancer tumor characteristics. Eur J Radiol 2006; 60: 398-404. (PMID: 17030108) [Crossref]

19. Verma R, Mathur R, Raikwar R, Kaushal M, Miishra H, Shukla R, et al. Comparison of clinical assessment, mammography, and ultrasound in pre-operative estimation of primary breast-cancer size: a practical approach. Internet J Surg 2008; 16: 12. [Crossref]

20. Boetes C, Mus R, Holland R, Barentsz JO, Strijk SP, Wobbes T. Breast tumors: comparative accuracy of MR imaging relative to mammography and US for demonstrating extent. Radiology 1995; 197: 743-747. (PMID: 7480749) [Crossref]

21. Gruber IV, Rueckert M, Kagan KO, Staebler A, Siegmann KC, Hartkopf A. Measurement of tumour size with mammography, sonography and magnetic resonance imaging as compared to histological tumour size in primary breast cancer. BMC Cancer 2013; 13: 328. (PMID: 23826951) [Crossref]

22. Ramirez SI, Scholle M, Buckmaster J, Paley RH, Kowdley GC. Breast cancer tumor size assessment with mammography, ultrasonography, and magnetic resonance imaging at a community based multidisciplinary breast center. Am Surg 2012; 78: 440-446. (PMID: 22472402) [Crossref]

23. D’Orsi CJ, Sickles EA, Mendelson EB, Morris EA. ACR BI-RADS Atlas, Breast Imaging Reporting and Data System. 5th ed. Reston, VA; American College of Radiology: 2013.

24. Morris EA. Diagnostic breast MR imaging: current status and future directions. Radiol Clin North Am 2007; 45: 863-880. (PMID: 17888774) [Crossref]

25. Ebili HO, Oluwasola AO, Olopade OI. Molecular subtypes of breast cancer, Personalized Management of Breast Cancer, Holloway, T.L., Jatoi, I., Eds.; Future Medicine Ltd.: London, UK, 2014; pp. 20-33 [Crossref]

26. Corp I. IBM SPSS statistics for windows, version 22.0, IBM Corp Armonk, NY. 2014. Available from: https://www.ibm.com/support/ pages/how-cite-ibm-spss-statistics-or-earlier-versions-spss

27. Grimsby GM, Gray R, Dueck A, Carpenter S, Stucky CC, Aspey H. Is there concordance of invasive breast cancer pathologic tumor size with magnetic resonance imaging?. Am J Surg 2009; 198: 500-504. (PMID: 19800455) [Crossref]

28. Onesti JK, Mangus BE, Helmer SD, Osland JS. Breast cancer tumor size: correlation between magnetic resonance imaging and pathology measurements. Am J Surg 2008; 196: 844-850. (PMID: 19095098) [Crossref]

29. Luparia A, Mariscotti G, Durando M, Ciatto S, Bosco D, Campanino PP. Accuracy of tumour size assessment in the preoperative staging of breast cancer: comparison of digital mammography, tomosynthesis, ultrasound and MRI. Radiol Med 2013; 118: 1119-1136. (PMID: 23801389) [Crossref]

30. Lai HW, Chen DR, Wu YC, Chen CJ, Lee CW, Kuo SJ. Comparison of the diagnostic accuracy of magnetic resonance imaging with sonography in the prediction of breast cancer tumor size: a concordance analysis with histopathologically determined tumor size. Ann Surg Oncol 2015; 22 : 3816-3823. (PMID: 25707494) [Crossref]

31. Alikhassi A, Omranipour R, Shahriyaran S, Hadji M, Abdi A, Alikhassy Z. Correlation between imaging and pathologic measurement of breast cancer tumor size. Arch Breast Cancer 2015; 2: 64-68. [Crossref]

32. Stein RG, Wollschläger D, Kreienberg R, Janni W, Wischnewsky M, Diessner J. The impact of breast cancer biological subtyping on tumor size assessment by ultrasound and mammography-a retrospective multicenter cohort study of 6543 primary breast cancer patients. BMC Cancer 2016; 16: 459. (PMID: 27411945) [Crossref] 


\section{Azhdeh et al. Accurate Estimation of Breast Tumor Size}

33. Rominger M, Berg D, Frauenfelder T, Ramaswamy A, Timmesfeld N. Which factors influence MRI-pathology concordance of tumour size measurements in breast cancer?. Eur Radiol 2016; 26: 1457-1465. (PMID: 26268905) [Crossref]

34. Baek JE, Kim SH, Lee AW. Background parenchymal enhancement in breast MRIs of breast cancer patients: Impact on tumor size estimation. Eur J Radiol 2014; 83: 1356-1362. (PMID: 24882786) [Crossref]
35. França LKL, Bitencourt AGV, Paiva HLS, Silva CB, Pereira NP, Paludo J. Role of magnetic resonance imaging in the planning of breast cancer treatment strategies: comparison with conventional imaging techniques. Radiol Bras 2017; 50: 76-81. (PMID: 28428649) [Crossref] 